\title{
EVS24 \\ Stavanger, Norway, May 13-16, 2009 \\ Charging Stations for Urban Settings the design of a product platform for electric vehicle infrastructure in Dutch cities
}

\author{
Hatton, C.E. ${ }^{1}$, Beella, S.K., Brezet, J.C., Wijnia, Y.C. ${ }^{2}$ \\ ${ }^{I}$ Delft University of Technology, Faculty of Industrial Design Engineering, \\ Landbergstraat 152628 CE Delft, the Netherlands, chandlerh@gmail.com \\ ${ }^{2}$ Delft University of Technology, Faculty of Technology, Policy and Management, \\ Jaffalaan5 2628 BX Delft, the Netherlands
}

\begin{abstract}
This paper reflects the essential role of supportive infrastructure in the mass implementation of electric drive vehicle technology. A focus is placed on the development of comprehensive systems that provide efficient and diverse recharging solutions for vehicle drivers. Mass adoption of electric mobility will reflect advances in the vehicles themselves, their infrastructural networks, and their position in the automotive market. Progress in these areas is highly related, and mandates coordinated design efforts. This paper discusses current problem areas, ongoing developments, and future trends in the design and development of charging systems for battery electric vehicles. The application of infrastructures that are simple, familiar, and context-sensitive is essential to promote consumer confidence and thereby enable successful market penetration. Specific requirements and conditions for the design of infrastructure systems for electric mobility are presented.
\end{abstract}

Keywords: battery charge, conductive charger, electric vehicle, fast charge, infrastructure

\section{Introduction}

Electric vehicles $(E V s)^{1}$ are known for their advantages as local, emission free, energy efficient, and noiseless means of transport. Still, electric mobility has never matured in the automotive market and remains in the shadow of the internal combustion engine. EV penetration is dependent on three primary factors: the specifications and capabilities of the vehicles

\footnotetext{
${ }^{1}$ This paper focuses specifically on infrastructures for battery electric vehicles. Other vehicles that utilize electro motors, including fuel cell vehicles and hybrid electric vehicles are not treated in this text, and the term "EV" will generally refer to all-electric vehicles.
}

(speed, torque, handling), the action radius and availability of recharging facilities, and the placement of EVs in the automotive market [1-3]. These three issues are highly interconnected and have contributed to a seemingly endless delay in market implementation.

Whereas the design of an EV is relatively simple, the issue of an EV infrastructure is complex and system dependent. Recently, the development of charging networks based on competitive business models has become a starting point for the implementation process, subsequently renewing interest in the development of EVs $[4,5]$. In the city of Rotterdam, promotion of EVs has started with pilot infrastructure installation projects, 
followed by demonstrations of electric two wheelers and delivery vans [6].

Charging patterns and available charging infrastructure have a large impact on the way in which people use EVs and consequently impact market penetration. This paper endeavours to develop the requirements and conditions that will contribute to the realization of a product platform for EV charging. As a starting point, the consumer base and potential penetration scenarios are discussed. Thereafter, several methods of vehicle charging are introduced, with a focus placed on techniques for standard and rapid conduction charging. Implications for the design of charging systems are presented in Section 4.

\subsection{Characteristics of EV systems}

As a system, drive train of a battery electric vehicle is simpler than that of an internal combustion engine (ICE) vehicle because it consists of only three components: an electro motor, a controller, and a battery. Electro motors can reach efficiencies of up to $90 \%$ [7]. Added benefits of regenerative breaking and torque handling make EVs especially well suited for stop-and-go conditions.

EV systems comprise two primary elements: the vehicle itself, and the equipment for connecting it to the electricity grid network. EV characteristics are therefore dependent on the size and chemistry of the vehicle battery and the corresponding charging patterns. Because vehicle batteries have a low energy density as compared to the liquid fuel of internal combustion vehicles, most EVs have a shorter action radius than their conventional counterparts. This limitation has hampered full-scale market implementation of EVs [8]. Yet "studies of vehicle use by a wide selection of the motoring public suggest that the typical range of an EV (100-130 km) is more than sufficient for the majority of journeys made in one day" [9]. Limited range in combination with minimal noise pollution and low vibration make EVs optimal for use in industrial complexes, densely built urban environments, and residential areas [10].

While EVs provide numerous advantages for specific driving conditions, they are often directly compared to traditional internal combustion vehicles designed for multi-purpose use. In such a comparison the shortcomings of EVs are marked: traditional vehicles have a larger driving range and come with a widespread refuelling and maintenance network. Thus, solutions related to the extension of the action radius, the reduction of the recharge time, and the accessibility of charging infrastructure must be sought.

\subsection{Marketing and product placement}

Technologies only survive in competitive markets with viable business cases and adequate public support. The key to success lies in the integration of technical strength and societal strength in the EV industry [10]. Chan et al. define 'societal integration' as governmental support, financing, and research and development efforts lead by academic institutions. These factors increase societal acceptance of and commitment to the implementation of electric mobility.

The California saga of late 90's illustrates the socio-technical obstacles that must be overcome to achieve large-scale penetration of zero-emission vehicles [11]. EVs are not new to the end-user; yet, they have historically existed in small numbers as niche vehicles. The mass implementation of EVs for personal transit is an innovative concept driven by contemporary issues of energy security, noise and air pollution, and global warming. The average personal vehicle driver is, of yet, unfamiliar with the EV. This presents a challenge in the product placement of electric mobility solutions for mass production.

Gärling and Thøgerson propose a two-phase strategy for the adoption of EVs [12]. The first phase is characterized by marketing directed toward the likely first adopters that make up approximately $2.5 \%$ of the market share, including public sector organizations, companies with an environmentally benevolent image, and multi-car households. The second phase is characterized popularization of the EV among a larger audience of individuals whose driving habits coincide with the capabilities of EVs. Successful adoption in the first phase of this model will contribute to but does not guarantee successful adoption in the second, as the two phases take place in different markets.

Given that energy technologies such as refineries and power stations require extremely large capital expenditures, the energy infrastructure built today will remain in operation for $30-40$ years [13]. This means that new infrastructure investments must target early adaptors as well as future users. Studies have indicated that customers are resistant to radical or seemingly untested technologies [13]. Furthermore, relative advantage, compatibility, triability, observability, and complexity are understood as contributors to innovation uptake [14]. Drivers must be presented with simple and 
robust systems if they are to embrace EV technology. Adventuresome early adaptors, who are not disheartened by the potential difficulties of new technologies, tend to be drawn to products because they identify with the product values; in the case of alternative mobility this equates to low emissions and energy efficiency [12].

The UK's Department for Business Enterprises and Regulatory Reform recently published a study on uptake scenarios for EVs [2]. The report emphasized the significance of triability in EV adoption routes and early interventions with captive fleets. Approaches for implementation can be driven by: Motor-industry lead technological-push, such as the Renault-Nissan participation in Project Better Place; a top-down approach using government regulation, such as the CARB legislation that was passed (and later repealed) in California in the late $90 \mathrm{~s}$; or a bottom-up approach whereby interested communities provide a market-pull. Successful efforts will probably adopt a combination of the three.

Once EV technology is in place, characterization of the product type choice factors that describe the typical EV driver will be possible. Type choice factors including travel attitudes, personality, and lifestyle will be relevant to transportation planners and policy-makers as well as vehicle manufacturers [15].

\section{EV infrastructure}

\subsection{Basic charging infrastructure}

It has long been understood that supportive infrastructure must be in place prior to the introduction of a new energy technology [13]. For this reason, contemporary installation of charging infrastructure is paramount for the imminent organization of large-scale EV systems. Widespread roll-out of electric mobility will require increased consumer confidence, necessitating that infrastructural development remain ahead of vehicle uptake [2]. Understanding vehicle systems and the opportunities infrastructural development affords must therefore be a top priority for proponents of electric mobility, as this knowledge will provide the basis for the realization of viable solutions for electric mobility.

\subsubsection{Charging equipment}

EV infrastructure comprises a network of charging stations capable of replenishing vehicle batteries. Conventionally, 'charging station' describes the physical location where EV batteries are recharged. This location is characterized by the presence of equipment to replenish depleted vehicle batteries as well as necessary parking space(s). Stations may accommodate one or more vehicles and may be intended for private or public use. In the case of conductive charging, the station itself may be subdivided into two primary components: the user interface and the socket that supplies electricity. A complete charging system includes various charging devices that recharge vehicle batteries in different manners to accommodate diverse driver needs.

An important distinction must be made between 'chargers' and 'charging stations', as the two terms cannot be used interchangeably. Battery chargers, or power processing units, are the devices that modulate the incoming current and voltage from the power supply in order to apply the correct charging algorithm for the battery chemistry. For example, lithium-ion batteries may be charged with a current-controlled, voltage-controlled program whereas nickel-metal-hydride batteries may utilize two consecutive constant-current stages. Different algorithms are required for different types of batteries as well as batteries of the same type that are at differing depths of depletion. In the case of domestic and standard charging, the charger is typically located onboard of the vehicle. However, in the case of rapid charging the charger is too large to be located on the vehicle itself and therefore finds place inside the charging station.

Charging can be carried out by applying a conductive or inductive current to the batteries or by swapping the depleted batteries with charged ones. The default method for vehicle battery charging is the use of a low current conductive connection between the battery and the power source, which is managed by either an onboard or off-board charger.

\subsubsection{Charging rates for vehicle batteries}

The long charge time associated with vehicle charging from conventional, low current outlets has resulted in an infrastructural network that is predominately based on domestic, overnight charging. However, as higher current connections become available and charge times are reduced and/or batteries and battery swapping procedures 
become universal, vehicle charging infrastructure will be extended beyond domestic settings.

The literature predicts that the EV driver will have access to several types of charging stations, providing various options for vehicle charging $[9,16]$. Among these options are overnight (default) vehicle charging, which requires a 6-8 hours for charge completion; standard (optimized) vehicle charging, which charges the vehicle as quickly as possible using an onboard charging unit; and accelerated vehicle charging, which charges the battery in as little as ten minutes $^{2}$.

\subsection{Rapid charging infrastructure}

According to a recent study conducted by the Netherlands Environmental Assessment Agency (PBL), the major disadvantages of electrified mobility solutions are the limited driving range of EVs and the practical problems associated with battery charging [17]. This handicap can be overcome by the use of accelerated battery recharging techniques.

In an early study of vehicle battery charging, Kahn emphasizes the desirability of rapid charging as a way to enable the use of EVs for applications beyond daily commuting [16]. In the Netherlands, the average daily commute is a mere $44 \mathrm{~km}$ [17]. Such a distance could easily be covered by the smallest EVs on the market. However, personal vehicles are also called upon for longer distance travel, albeit less frequently. PBL research suggests that the driving range of larger EVs must be at least $300 \mathrm{~km}$, to meet the needs of the $99 \%$ of the population that travel less than $300 \mathrm{~km}$ in a single day [17].

Indeed, so long as vehicle batteries lack the energy density capable of extending vehicle range to rival that of conventional combustion engine vehicles, a rapid charging solution is critical for EV acceptance among consumers.

\subsubsection{Rapid charging equipment}

For some battery chemistries it is possible to increase the rate at which the battery is charged

\footnotetext{
${ }^{2}$ Kahn suggests that standard charging should utilize an off-board charging unit capable of charging a battery pack in 2-3 hours. However, contemporary technology enables comparable charge times using onboard chargers. Further mention to standard charging will reference contemporary onboard charging units that charge the vehicle battery in as little time as possible.
}

by increasing the flow of current from the power source. This necessitates the use of a larger charger in order to mitigate larger AC or DC currents. The size of the charger needed for current regulation negates the possibility of providing rapid charging using onboard chargers. As a result, vehicles equipped with both default/standard- and rapidcharging capabilities require two separate charging outlets for their respective low and high-current connections ${ }^{3}$.

Proposed rapid conductive charging systems have received mixed reviews among industry professionals and academics. State of the art rapid charging processes can have adverse affects on vehicle batteries and should therefore be performed only when an immediate extension of vehicle range is necessary [18]. It is therefore recommended that the use of rapid charging be restricted to less than $5 \%$ of the total number charging cycles that a battery undergoes (if at all). Moreover, the effect of rapid vehicle charging on the electricity grid network remains unknown. Conversely, several recent studies have expressed optimism regarding the feasibility of rapid charging, indicating that the ability of various battery chemistries to accept a rapid influx of charge is likely to improve in coming years. Nevertheless, the high cost of installing rapid charging infrastructure and the difficulty associated with drawing large amounts of energy from electricity grid ensure that overnight and standard charging will remain the most common methods for vehicle charging.

An alternative method of quickly recharging EVs is to replace depleted batteries with a charged ones. This method is referred to as battery swapping. To date, very little literature has addressed the physical plausibility of battery swapping. However, battery and charging system manufacturers have identified several disadvantages, including the excess space and money required for the necessary surplus of batteries, the potential damage to batteries that may result from excessive swapping, the need for vehicle redesign to enable easy access to battery packs, and the implicit standardization necessary [19].

Efficient battery charging facilities are critically important because drivers are hesitant to invest in vehicles that potentially limit their personal

\footnotetext{
${ }^{3}$ Standard-charging is regulated by an onboard charger while rapid-charging is regulated by an off-board unit, which communicates with the onboard battery management system.
} 
mobility. Should rapid charging or battery swapping techniques be discarded in the development of a comprehensive vehicle infrastructure scheme, then appropriate amends must be made. Systems that opt for exclusive use of standard onboard charging systems must address documented consumer demand for simple, fast, and dependable infrastructure to extend the range of EVs beyond urban boundaries.

\subsubsection{Potential charging patterns}

Because of the uncertainties surrounding rapid charging technology, emphasis has been placed on default and standard charging scenarios. A concern is the logistical problem of accommodating default charging times in excess of six hours. It is feasible to use a 22 $\mathrm{kW}$ charging station to charge an EV battery in a little less than two hours. Standardization efforts are necessary to ensure that such charge times can be achieved by the vast majority of EVs. Charging stations capable of producing charge times that coincide with amount of time a car might be parked at a public lot enable the realization of a viable charging network for public locations.

With significant penetration rates for $\mathrm{EVs}$, such as the "mid-range" (12\% by 2030$)$ and "high-range" $(30 \%$ by 2030) scenarios proposed in the BERR study, pervasive charging infrastructure must be installed. This includes charging stations at all garage, street, and domestic parking locations.

Rapid charging stations will likely find place along highways and provincial roads. Because overnight and standard charging protocols will continue to be the primary method for battery recharging, few rapid charging stations will be necessary in the EV infrastructure.

A comprehensive vehicle infrastructure of the nature described above would enable the realization of EVs as a competitive form of personal mobility. The replacement of domestic charging facilities with standard and rapid charging stations should not be underestimated. The BERR report emphasizes the importance of public charging facilities for practical and peace-of-mind reasons, with rapid charging providing security for nervous potential users [2]. Several sources identify roadside battery recharging amenities as a critical step in the expansion of EVs beyond urban environments $[2,16]$. It is through the realization of an efficient and comprehensive infrastructure that EVs will be able to rival the reign of the internal combustion engine.

The affect that mass vehicle charging scenarios will have on the electricity grid network remains speculative. This topic is particularly interesting from the perspective of distributed energy systems [20]. It is certain that vehicle penetration will have consequences for the size and capacity of grid infrastructure.

\subsubsection{Charging technology meets vehicle design}

Initially, EVs were produced in small quantities and often retrofitted from conventional ICE vehicles. The state of the art continues to follow suit: the same ICE production models are reintroduced as EVs and vehicle modifications remain under the hood. Yet electric mobility solutions have a different typology with a different drive train and energy source. EVs will achieve maximum functionality if they are designed in accordance with the basic principles of $\mathrm{EV}$ technology. One of the fundamental characteristics of electric mobility that must be taken into account in vehicle design is the need to charge the vehicle batteries. Consideration of various charging scenarios, particularly rapid charging or battery swapping, during the vehicle design process will enable the development of vehicles that truly meet the needs of their users.

Technology can aid us in performing both simple and complex tasks. However, technology cannot aid us in determining the specific tasks to which it can best be applied. The designation of product specifications enables appropriate application of technologies. Rapid charging mandates specific system requirements, such as preferred battery chemistries, necessary loading capacity, and the need for cooling/ventilation. The development of a scenario in which rapid charging promotes market penetration of EVs will provide stimulus for the development of rapid battery charging systems. This action/reaction sequence can already be seen in the automotive industry, as many vehicle manufacturers are venturing into Lithium-ion battery development.

\section{Stimulating EV penetration}

The ability of the EV to move beyond its current niche market is dependent on vehicle specifications, availability and convenience of charging infrastructure, and market placement. 
The task of assigning vehicle specifications will be left to the vehicle manufacturer. The sections below summarize the need for proper marketing and the construction of supportive infrastructure.

\subsection{EV marketing}

A fast transition to electric mobility demands an increase in the competitiveness of EVs in the automotive market. Large-scale adoption will require understanding of the type choice characteristics of electric mobility users which will inform product branding and policy development. Favoured adoption routes should consider vehicle demonstration projects as an initial step toward mass deployment. EVs should not be improperly marketed to individuals whose driving habits do not coincide with the advantages of electric mobility. Finally, new EV drivers may be precautious, so triability should be emphasized.

\subsection{Rapid charging}

Regardless of the low frequency with which rapid charging systems are likely to be used, the installation of rapid charge facilities is an essential part of the development of a charging network because efficient charging protocols will enable EVs to break free of the niche market that they currently occupy.

Work towards the optimization of adequate and efficient vehicle infrastructure should concentrate on the ways in which vehicles and vehicle infrastructures can be redesigned to better accommodate the mobility needs of the EV driver. Continued research in the field of battery technology must be bolstered by contributions from designers and other stakeholders to identify opportunities for the improvement of the viability, economics, and usability of infrastructural systems.

\subsection{EV infrastructures}

It is understood that electric car drivers will have the luxury of selecting among a variety of recharging options [9]. Moreover, it is appreciated that rapid charging is highly desirable "because it allows people to use EVs to do things other than just commuting" [16]. Yet methods for designing a vehicle charging infrastructure that incorporates charging apparatuses with a variety of options for charging speed have not yet been elucidated. Requirements and conditions for the development of such systems are discussed in following section.

\section{The design of EV charging infrastructure}

\subsection{The need for a comprehensive infrastructure}

The realization of appropriate vehicle charging infrastructures will require collaborative design efforts involving a broad range of stakeholders [9]. Proposed systems must consider the roles of the suppliers, operators, customers and/or users.

To date, little work has been done to formalize the requirements for the design of infrastructure for electric mobility. Recently, Europe's Union of the Electricity Industry - Eurelectric has set out to standardize connections between vehicles and the power grid [21, 22]. These coordination efforts will contribute to international compatibility and may pave the way for infrastructural projects across Europe. Yet, the provision of standard electrical connections is only a part of the process of developing a supportive EV charging network. EV market research emphasizes the development of infrastructural systems that are simple, familiar, and context-sensitive to boost consumer confidence and enable successful market penetration [13]. This section delves into the specific requirements and conditions for designing infrastructures for electric mobility.

\subsection{Definition of two design tasks}

The realization of appropriate EV infrastructure mandates two primary design efforts: system-level design (i.e. design of the operational structure of vehicle charging systems) and product-level design (i.e. design of charging apparatuses). Current research efforts typically inform development on one or the other of these scales. System-level research addresses topics like the ability of the energy grid to accept high penetration of EVs [9]; opportunities for smart charging and Vehicle-toGrid (V2G) systems [23-25]; life cycle analyses of EV infrastructures [26]; and the business models that support economic feasibility. Other research focuses on technical issues, such as charging equipment and control strategies $[16,27])$; usage issues, such as the requirements for charging stations [28]; and issues associated with battery technology. This body of research can be 
characterized as investigations on the scale of the charging apparatus.

The intimate relationship between the developments taking place on these two scales underscores the necessary synchronization of design efforts. It is therefore proposed that these processes are carried out in parallel, with systemlevel solutions contributing to developments on the scale of the apparatus and vice versa. Figure 1 depicts system-level and product-level design efforts as parallel and overlapping trajectories System-level design is portrayed as a collaborative effort involving all stakeholders, while product-level design involves only a select few. External inputs remain relevant throughout the design process, which is particularly important because the field of electric mobility is developing at an extreme rate. Specific inputs for these parallel processes are elaborated in the following section.

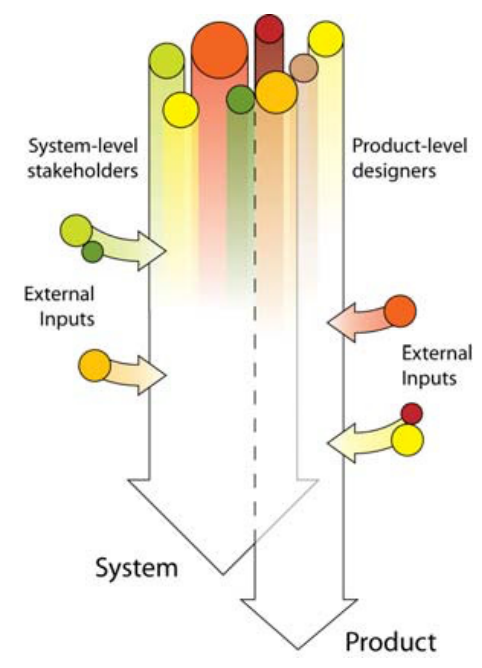

Figure 1: Parallel design process for development on the system-level and product-level.

\subsection{Appropriate design inputs}

Research presented in this section was informed by interactions with various parties involved in the organization of charging infrastructure in The Netherlands; stakeholders in the implementation of electric mobility programs were consulted in personal interviews and group workshops. These discussions lead to recommendations for infrastructural development projects and the formulation of a set of product requirements for the design of EV charging stations. The requirements and were tested with a group of four of Dutch designers during three days of design sessions. These sessions elucidated necessary inputs for the development of both infrastructural systems and charging apparatuses.

\subsubsection{System-level inputs}

The cooperation of a broad range of stakeholders will advance the development of appropriate infrastructures to support electric mobility, as diverse stakeholder perspectives will directly contribute to the realization of a charging system that benefits all parties concerned with the set-up and operation of electric mobility systems [9].

Inputs for system-level design will come in the form of statistics, projections, and scenarios. Region or Nation-specific analyses of EV driving and vehicle charging opportunities form a basis for the development of specific usage scenarios for EVs and corresponding infrastructures. National studies, such as the BERR investigation into the scope of a switch to electric mobility in the UK and the PBL study of system options for electric mobility in The Netherlands aid in predicting system capacities and identifying target users [2, 17]. Studies such as these can be linked to statistical data on vehicle ownership and driving habits to arrive at quantifiable projections for EV use in the coming decades. Stakeholder coalitions may work from these projections, as well as from the contributions of technical research in the field of electric mobility to organize operational infrastructure systems.

It should be stressed that these systems must be calibrated for regional characteristics including urban, geographic, and cultural contexts. Moreover, the orchestration of the electrical utilities networks will have a large effect on the type of system-model appropriate for $\mathrm{EV}$ infrastructure.

It is likely that emergent systems will mandate some degree of experimentation and revision prior to full-scale implementation. The various international EV infrastructure projects currently underway confirm the significance of pilot and demonstration projects, which "will be critical to address the questions of all stakeholders involved in PHEV and EV" [2]. Early versions of EV infrastructural systems should be tested in a controlled manner to ensure that they are suitable for large-scale implementation.

\subsubsection{Apparatus-level inputs}

Because electric mobility draws on new energy technologies, there is a need for dynamic 
infrastructural systems that can be modified in accordance with rapidly developing technologies and business process. "It is most important to develop an infrastructure that is flexible enough to benefit from technological improvements which may take places as the technology matures"[16]. The emergent vehicle charging infrastructure will be closely related to smart grid networks, the development of which requires "flexible design, agility, and improvisation necessitated by frequent and dramatic changes that are ill-suited for traditional utility-style projects" [29].

It follows that EV charging infrastructures must provide flexibility to enable further optimization of the technologies, interfaces, and business models that govern the systems. The need for flexibility, usability, and compatibility has informed the generation of the comprehensive set of requirements for the design of EV charging apparatuses provided in the following section.

\subsection{Requirements for the design of vehicle battery charging stations}

This set of design requirements has been constructed for the various parties involved in the development of vehicle charging infrastructure in The Netherlands, with a specific focus on the product designer. The design requirements have been devised with current state-of-the-art technology in mind to facilitate immediate infrastructural development. Recent discoveries at the cusp of applicability have been included, but design proposals based on these technologies should be utilized with discretion.

The list has have been divided into eight categories: logistics, functionality, interface, form, aesthetics and semantics, safety, durability and maintenance, and production and end of life. Specific requirements fall under the category that best suits them. Designers should always consider the entire list of design requirements rather than a single section.

Within each category, the requirements are presented in order of priority. Some requirements are listed under the heading "demands", while others are listed under the heading "wishes". One difference between demands and wishes is the degree to which the requirements are measurable. Demands are quantitative and provide finite values and definitive conditions. By contrast, wishes are qualitative and identify factors that should be minimized or maximized. Additionally, wishes provide the designer with a greater degree of flexibility. If wishes are not met, the product will likely work; if wishes are met the product will likely work well. Designers should strive to meet all of the demands and wishes.

\subsubsection{Logistics}

Demands:

L-1. The charging system must support basic charging for all types of plug-in electric vehicles. Either default charging or standard charging at currents below $16 \mathrm{~A}$ must be possible. For basic charging, communication between the vehicle and the charging station must not be required for use of the charging station.

L-2. EU regulation regarding the standardization of plugs for electric vehicle charging stations is expected in the coming years. All plugs and sockets must therefore be accessible for upgrades.

L-3. The charging system must have the capacity to charge vehicle batteries in as little time as possible using a rapid or standard charging profile. Charging at currents greater than $64 \mathrm{~A}$, rapid charging, requires an off-board charger that can apply appropriate charging algorithms.

L-4. Stations that offer rapid vehicle charging must provide all cables and plugs necessary, as this equipment is too heavy and cumbersome to be efficiently transported by vehicle drivers.

L-5. Due to acute differences in production costs and requisite traffic management, each charging station must be dedicated to either default, standard, or rapid vehicle charging. Together, these different types of stations make up the range of charging stations within the charging system.

L-6. Plugs and sockets must be standardized within the charging system.

L-7. Charging stations must be self-service and available for use 24 hours a day, 7 days a week.

Wishes:

L-8. Parking spaces for vehicle charging must be located as close to the interface as possible.

L-9. Charging stations must be as standardized as possible; distinct surroundings should not necessitate a unique design. 
a. Stations for default and standard vehicle charging should be appropriate for or adaptable to various urban environments, including curbside, parking lot, and garage locations.

b. Stations for rapid vehicle charging should be appropriate for or adaptable to both highway and urban locations.

L-10. If vehicle charging facilities are not provided at all parking locations within the city or region serviced by the charging system, then parking spaces at charging stations should be dedicated to vehicle charging only.

L-11. Charging stations should accommodate as many vehicles as possible.

L-12. Charging stations should not affect normal traffic flow.

\subsubsection{Functionality}

Demands:

F-1. All cables and plugs provided by the charging stations must comply with IEC standards.

F-2. The charging terminal jacks must not provide electrical current when the charging station is not connected to a vehicle and/or a charging sequence has not been initiated.

F-3. Charging must take place in a well ventilated area, as batteries may gas if improperly or excessively charged.

F-4. For rapid charging using an off-board charger, the specifications for ventilation and cooling recommended by the charger supplier must be met.

F-5. Each outlet must be individually metered.

F-6. A digital- or hard-copy of accounting processes (including proof of transaction) must be available to users.

F-7. The charging station must be protected against the misappropriation of electricity by unauthorized persons.

F-8. Once charging has commenced and electrical current is flowing from the charging station to the vehicle, it must not be possible for unauthorized persons to disturb or adjourn the charging process. Interference with vehicle charging must be restricted to the initiator of the process and maintenance/safety officials.

F-9. It must be possible for experienced users to set up the charging station to begin charging in less than 30 seconds. This excludes the time required for parking etc.

F-10. Sockets must have bi-directional metering to enable a future transition to a smart grid system. When and if the smart grid system is established, remote monitoring by grid operators must be possible.

F-11. If a comprehensive charging system is installed, then the specifications posed by MEE Nederland must be met.

Wishes:

F-12. It should not be necessary to use both hands to operate a charging station.

F-13. A user should never have to lift or maneuver components weighing more than $3 \mathrm{~kg}$.

F-14. The position of the sockets should be suitable for people with a wrist height of 688-895 mm.

F-15. For vehicle security, it should be possible to lock two-wheelers to a fixed object during charging. The lock may be supplied either on-site or by the user.

F-16. Any modifications to the vehicle itself required for use of the charging system should be completely reversible.

\subsubsection{Interface}

Demands:

I-1. Individuals who understand basic Dutch and English must be able to operate the interface.

I-2. The position of the user interface must be suitable for people with an eye height of 1460-1839 mm.

I-3. A Dutch person of 18-65 years of age with an average level of education must understand how to operate the charging station within three minutes when using the charging system for the first time.

I-4. Users must get immediate feedback from every interaction with the interface. This feedback may be tactile, audio, visual, etc.

I-5. Interactive instructions for vehicle charging must be provided: during the setup process, the interface must respond to 
user actions and provide corresponding instructions.

I-6. For rapid charging stations and standard charging stations that supply a current greater than $16 \mathrm{~A}$, information must be provided during the charging process, including:

a. Information regarding the charging status (i.e. 'charging', 'charged', 'service required', etc.).

b. State of charge (SOC) of the vehicle battery.

c. Time remaining until charge completion.

...This requires a feedback loop between the onboard battery management unit and the charger.

I-7. For rapid charging stations and standard charging stations that supply a current greater than $16 \mathrm{~A}$, Customers must be able to input specific charging parameters. A choice between the desired charge time, state of charge, and cost must be available.

I-8. The charging system must include a remote online interface with the following features:

a. Map of charging station locations.

b. Trip planning.

c. Map of the availability of charging stations.

d. Current electricity rates.

Wishes

I-9. Prior membership should not be required for use of the charging stations.

I-10. If the interface provides membershipbased features, then customers should be uniquely identified by means of a login, key, pass, etc.

I-11. The remote online interface presented in designer requirement I-8 should provide the following features:

e. User profiling and statistics.

f. Instruction manuals.

g. Customer support.

\subsubsection{Form}

Demands:

O-1. For security, charging stations must be anchored to an object or surface in the surrounding environment.
O-2. Public stations must be recognizable from a distance of $100 \mathrm{~m}$.

Wishes:

O-3. The form of charging stations and their components should be indicative of the underlying function.

\subsubsection{Aesthetics and Semantics}

Demands:

A-1. The range of charging stations within a charging network must have a consistent aesthetic.

A-2. Charging stations must be visually compatible with the urban environments that they will occupy.

Wishes:

A-3. The user interface should communicate that the station it is simple to use.

A-4. The charging stations should tell the story of electric vehicle charging.

\subsubsection{Safety}

Demands:

S-1. The risk of electrical shock during charging station operation must be eliminated. It must not be physically possible to make electrical connections that are dangerous to persons or properties.

S-2. The device must meet CE-marking standards.

S-3. All electrical connections must achieve a watertight rating of IP54.

Wishes:

S-4. Any components spanning the distance between vehicles and charging station sockets should not be hazardous to pedestrians, cyclists, or drivers.

S-5. Pinch points and sharp edges that could potentially harm users, their clothing, or their belongings should be minimized.

S-6. The software system should minimize the risk of fraudulent behavior.

\subsubsection{Durability and Maintenance}

Demands:

D-1. Charging stations and their sub-elements must be able to withstand the weather typical to the Netherlands. This includes: a. A working temperature range of $-10^{\circ}$ $\mathrm{C}$ to $30^{\circ} \mathrm{C}$. 
b. An outdoor temperature range of -30 ${ }^{\circ} \mathrm{C}$ to $40{ }^{\circ} \mathrm{C}$ (the station does not necessarily need to operate at extreme temperatures, but no maintenance should required).

c. Wind speeds of up to $150 \mathrm{~km} / \mathrm{h}$.

d. Daily exposure to UV light.

D-2. The charging station must not degrade or corrode as a result of exposure to the chemical agents and other hazardous materials commonly found in urban environments.

D-3. Easy access for maintenance work must be provided, and access to the interior of the machine must be restricted to maintenance officials.

D-4. A statistical record of past charging events must be maintained to enable trouble shooting in the case of system malfunction.

D-5. The interface must accommodate future software modifications.

Wishes:

D-6. The charging stations should not be vulnerable to acts of vandalism.

D-7. Stations should be protected from vehicular collisions.

D-8. The charging station should not be easily damaged by the wear and tear of everyday use.

\subsubsection{Production and End of Life}

Demands:

$\mathrm{P}-1$. Once the charging station has reached the end of its functional life, it must be possible to disassemble the station components for disposal, recycling, or reuse.

Wishes:

P-2. The materials and production methods employed in the manufacturing process should be minimally harmful to the environment.

\section{Conclusions}

The establishment of comprehensive infrastructures for vehicle charging is prerequisite to the realization of electric mobility. Suitable infrastructure should provide charging opportunities at a variety of rates, to enable drivers to charge in the manner that they find convent. System designers should consider: overnight (default) vehicle charging using traditional low-current power connections; standard (optimized) charging for typical, everyday charging needs; and rapid charging methods that facilitate the extension of vehicle range. Emphasis should be placed on public charging amenities that enable the reduction of charging times.

Stakeholders must work cooperatively to establish infrastructural systems capable of overcoming the socio-technical barriers that currently impede the implementation of electric mobility. These systems should support the most plausible adoption routes for EVs; robust, state of the art solutions should be favoured over technological stretches. Early EV infrastructure projects should be tested in a controlled manner before they are deployed at the large-scale.

The proposed parallel design process for infrastructural development will aid collaborative design efforts in the specification of innovative and economically plausible systems. Product-level solutions should be informed by developments on the system level and by technical knowledge provided by experts in battery, electricity grid, and vehicle technology. Knowledge in these fields will also benefit from early implementation projects. Demonstrations may investigate grid capacity and energy storage issues as well as the relationship between vehicle charging and distributed energy systems. Cooperation of the automotive industry will be necessary to address issues such as plug standardization, communication technology, and the use of vehicle coding for authorization techniques. 


\section{References}

[1] P. J. Fontaine, "Shortening the Path to Energy Independence: A Policy Agenda to Commercialize Battery-Electric Vehicles," The Electricity Journal, vol. 21, pp. 22-42, July 2008.

[2] BERR, "Investigation into the Scope for the Transport Sector to Switch to Electric Vehicles and Plug-in Hybrid Vehicles," October 2008.

[3] WEC, "Transport Technologies and Policy Scenarios to 2050" 2007.

[4] Project Better Place, http://www.betterplace.com/, accessed on 2009-01-06.

[5] RWE, http://www.rwe.com/, accessed on 2009-01-06.

[6] NRGspot, http://nrgspot.eneco.nl/, accessed on 2009-01-30.

[7] H. L. MacLean and L. B. Lave, "Evaluating automobile fuel/propulsion system technologies" Progress in Energy and Combustion Science, vol. 29, pp. 1-69, 2003.

[8] K. Jorgensen, "Technologies for Electric, Hybrid and Hydrogen Vehicles: Electricity from Renewable Energy Sources in Transport" Utilities Policy, vol. 16, no. 2 pp. 72-79, 2008.

[9] R. Webster, "Can the electricity distribution network cope with an influx of electric vehicles?" Journal of Power Sources, vol. 80 , pp. 217-225, 1999.

[10] C. C. Chan and Y. S. Wong, "Electric Vehicles Charge Forward," in IEEE power \& energy magazine, 2004.

[11] R. van den Hoed, "Driving fuel cell vehicles: how established industries react to radical technologies" Delft University of Technology, Delft, The Netherlands, Doctoral dissertation, 2004.

[12] A. Gärling and J. Thøgersen, "Marketing of electric vehicles" Business Strategy and the Environment, vol. 10, pp. 53-65, 2001.

[13] B. K. Sovacool and R. F. Hirsh, "Beyond batteries: An examination of the benefits and barriers to plug-in hybrid electric vehicles
(PHEVs) and a vehicle-to-grid (V2G) transition" Energy Policy, vol. 37, pp. 10951103,2009

[14] E. M. Rogers and F. F. Shoemaker, "Communication of innovations: a crosscultural approach" New York: Free Press, 1971.

[15] S. Choo and P. L. Mokhtarian, "What type of vehicle do people drive? The role of attitude and lifestyle in influencing vehicle type choice" Transportation Research Part A: Policy and Practice, vol. 38, pp. 201-222, 2004 .

[16] I. A. Khan, "Battery chargers for electric and hybrid vehicles" in Power Electronics in Transportation, 1994. Proceedings, 1994, pp. 103-112.

[17] Netherlands Environmental Assessment Agency (PBL), "Driving Electric Vehicles: Evaluation of transitions on the basis of system options" Bilthoven, The Netherlands, January, 2009.

[18] S. S. Zhang, "The effect of the charging protocol on the cycle life of a Li-ion battery" Journal of Power Sources, vol. 161, pp. 13851391, 2006.

[19] AeroVironment, http://avinc.com/, accessed on 2008-12-09.

[20] M. Mirata, "Industrail Symbiosis: A tool for more sustainable regions?" IIIEE Lund University, Lund, Sweden, Doctoral dissertation, 2005.

[21] Eurelectric, http://www.eurelectric.org/, accessed on 2009-03-21.

[22] Eurelectric, "Electric Vehicles," BrusselsNovember 2008.

[23] H. Lund and W. Kempton, "Integration of renewable energy into the transport and electricity sectors through V2G" Energy Policy, vol. 36, pp. 3578-3587, 2008.

[24] W. Kempton and J. Tomic, "Vehicle-to-grid power fundamentals: Calculating capacity and net revenue" Journal of Power Sources, vol. 144, pp. 268-279, 2005.

[25] W. Kempton and J. Tomic, "Vehicle-to-grid power implementation: From stabilizing the grid to supporting large-scale renewable 
energy" Journal of Power Sources, vol. 144, pp. 280-294, 2005.

[26] K. Nansai, S. Tohno, M. Kono, M. Kasahara, and Y. Moriguchi, "Life-cycle analysis of charging infrastructure for electric vehicles" Applied Energy, vol. 70, pp. 251-265, 2001.

[27] J. Zhenhua and R. A. Dougal, "Control strategies for active power sharing in a fuelcell-powered battery-charging station" Industry Applications, IEEE Transactions on, vol. 40, pp. 917-924, 2004.

[28] H. Weiguo and D. Chengang "The Charging Model of the Plug-in Electric Vehicles and the Design of a Universal Charging Station for the Electric Vehicles," 2007.

[29] A. Vojdani, "Smart Integration" Power and Energy Magazine, IEEE, vol. 6, pp. 71-79, 2008 .

\section{Authors}

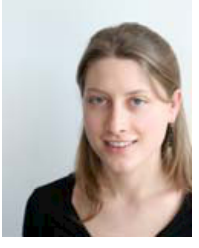

Chandler Hatton, MSc

Delft Design Institute

Faculty of Industrial Design

Delft University of Technology

C.E.Hatton@student.tudelft.nl

Chandler Hatton has recently completed her masters degree in Industrial Design Engineering at the Delft University of Technology. Her thesis work addressed the role of product designers in the development of electric vehicle charging systems, presenting guidelines for infrastructural development projects in the Netherlands. Chandler holds dual bachelors degrees from the Massachusetts Institute of Technology in architecture and mechanical engineering.

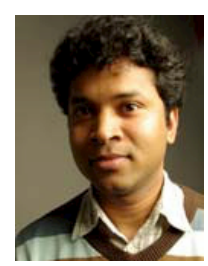

Satish Kumar Beella, MSc

Delft Design Institute

Faculty of Industrial Design

Delft University of Technology

S.K.Beella@tudelft.nl

Ir. Satish kumar Beella holds his masters degree in Industrial Design from Indian Institute of Technology Delhi, India. Satish's present research activities are mainly based on sustainable transportation innovations. From 2005, he is busy with design based $\mathrm{PhD}$ research at Delft University of Technology concerning of alternatives for urban car transport and solutions for chain mobility options. He has presently responsible role for alternative mobility development in innovative projects like 'D-incert' and 'Cradle to Cradle Islands'. He also involved in ongoing design and developments activities for sustainable Fryslân, which includes Terschelling baggage transportation system and innovative public transport options.

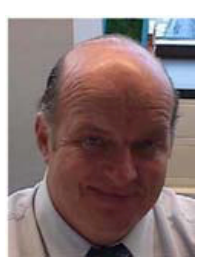

Prof. Dr. J.C. Brezet

Design for Sustainability

Faculty of Industrial Design

Delft University of Technology

J.C.Brezet@tudelft.nl

Prof. Dr. J.C. Brezet, since 1992 is heading the Design for Sustainability research program at the Faculty of Industrial Design Engineering at the Delft University of Technology in the Netherlands. In addition he is part-time professor at the International Institute for Industrial Environmental Econ-omics of the Lund University in Sweden. Besides he is a visiting lecturer at several universities in Europe. His current research is focused on Design for Sustainability, Sustainable Energy Technologies, Sustainable Regional Development and Sustainable Product Service Systems.

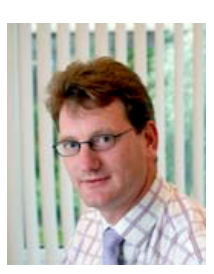

Ype Wijnia, MSc

Next Generation Infrastructures Faculty of Technology Policy and Management Delft University of Technology y.c.wijnia@d-cision.nl

Ype Wijnia has in-depth knowledge and experience in the area of Risk and Asset Management of energy infrastructures. As a PhD student at the faculty Technology, Policy and Management of Delft University of Technology he studies the optimal design and organisation of decision making for asset intensive industries, specializing on the energy sector. From 1999 Ype had, as risk manager, a leading role in the process and organisation design of the Asset Management department of Enexis, a large Dutch energy distribution company. He performed some world class studies, like the design of a safety indicator for the gas grid and the long term replacement policy for network assets. Ype is currently managing director of D-Cision B.V., a consultancy firm established in Zwolle, the Netherlands. 